\title{
Costo-efectividad de las estatinas para el tratamiento de dislipidemia en Colombia
}

\section{Cost-effectiveness of statins for the treatment of dyslipidemia in Colombia}

\author{
Diego Rosselli, Natalia Castaño, Jair-Alberto Arciniegas, \\ Ángel A. García, Óscar-Mauricio Muñoz, Carlos Gómez-Restrepo • \\ BogotÁ, D.C. (Colombia)
}

\section{Resumen}

Objetivo: determinar la relación de costo-efectividad relativa de las estatinas entre sí en pacientes adultos con dislipidemia con predominio de hipercolesterolemia para la prevención de eventos cardiocerebrovasculares, en Colombia, desde la perspectiva del sistema de salud colombiano.

Métodos: se construyó un modelo de Markov con años de vida ajustados por calidad (AVAC) ganados como desenlace principal, tasa de descuento de $3 \%$ tanto para costos como desenlaces, y cinco años de horizonte temporal. Probabilidades y costos se extrajeron de la literatura y de fuentes oficiales del país, respectivamente. El umbral de costo-efectividad fue tres veces el PIB per cápita de 2012. Se realizaron análisis de sensibilidad univariados, probabilísticos, umbral y curva de aceptabilidad.

Resultados: el costo promedio del tratamiento con estatinas para un paciente con dislipidemia en cinco años es de \$3 472 733. La ganancia en AVAC en rosuvastatina fue mayor con respecto a las otras estatinas; sin embargo, es la estrategia más costosa. La atorvastatina, que resultó segunda, es la más costo-efectiva.

Conclusión: la intervención más costo-efectiva para pacientes con dislipidemia con predominio de hipercolesterolemia e indicación de manejo farmacológico con terapia moderada y alta con estatinas en Colombia es la atorvastatina. (Acta Med Colomb 2015; 40: 118-124).

Palabras clave: análisis costo-efectividad; estatinas, dislipidemia, colombia, análisis económico.

\begin{abstract}
Objective: to determine the relative cost-effectiveness among statins in adult patients with dyslipidemia with predominant hypercholesterolemia to prevent cardiocerebrovascular events in Colombia, from the perspective of Colombian health system.

Methods: a Markov model with quality-adjusted life years (QALY) gained as the main outcome, discount rate of $3 \%$ for both costs and outcomes, and five-year time horizon was built. Probabilities and costs were extracted from the literature and from official sources in the country, respectively. The threshold for cost-effectiveness was three times the 2012 per capita GDP. Univariate probabilistic, sensitivity analysis, threshold and acceptability curve were performed.

Results: the average cost of statin therapy for a patient with dyslipidemia in five years is $\$ 3472733$. The QALY gain was higher in rosuvastatin compared to other statins, however, it is the most costly strategy. Atorvastatin, which was second, is the most cost-effective.

Conclusion: the most cost-effective intervention for patients with dyslipidemia with predominant hypercholesterolemia and indication of pharmacological management with moderate and high statin therapy in Colombia is atorvastatin. (Acta Med Colomb 2015; 40: 118-124).
\end{abstract}

Keywords: cost-effectiveness analysis; statins, dyslipidemia, Colombia, economic analysis.
Dr. Diego Rosselli: Neurólogo. Profesor Asociado, Departamento de Epidemiología Clínica y Bioestadística; Sres. Natalia Castaño y JairAlberto Arciniegas: Economistas, Asistentes de Investigación, Departamento de Epidemiología Clínica y Bioestadística; Dr. Ángel A. García: Cardiólogo Departamento de Medicina Interna y Hospital Universitario San Ignacio; Dr. ÓscarMauricio Muñoz: Internista, Departamento de Medicina Interna y Hospital Universitario San Ignacio; Dr. Carlos Gómez-Restrepo: Psiquiatra Epidemiólogo. Director Departamento de Epidemiología Clínica y Bioestadística. Facultad de Medicina, Pontificia Universidad Javeriana. Bogotá, D.C. (Colombia).

Correspondencia. Dr. Diego Rosselli. Bogotá, D.C. (Colombia).

E-mail: diego.rosselli@gmail.com

Recibido: 28/VI/2014 Aceptado: 13/II/2015

\section{Introducción}

En Colombia, la enfermedad cardiovascular es la primera causa de muerte y su participación porcentual aumenta cada año (1). El enfoque de riesgo múltiple se ha convertido en la estrategia de promoción y prevención que busca reducir la incidencia de enfermedades cardiocerebrovasculares (2). 
Dada la relación directa entre las concentraciones séricas de colesterol y el riesgo de enfermedad coronaria (3-6) la detección y el tratamiento de los trastornos del perfil lipídico se han convertido en un objetivo fundamental (7-9). Las estatinas constituyen la primera elección farmacológica para la disminución del colesterol LDL (10) dada la evidencia de que su uso reduce la tasa de mortalidad y los eventos vasculares $(7,11)$.

Por esta razón, entre los temas prioritarios para la elaboración de las Guías de Atención Integral que viene desarrollando el Ministerio de Salud y Protección Social conjuntamente con Colciencias, se seleccionó la atención de dislipidemias. Aunque la terapia con estatinas no clasifica como de "alto costo", la prevalencia de la condición hace que su impacto económico sea significativo. Se tienen, además, diferentes opciones terapéuticas con estudios de buena calidad, por lo que el grupo desarrollador de la guía le dio prioridad a la pregunta económica que motivó esta evaluación.

Es así que esta evaluación económica busca determinar la relación de costo-efectividad de las estatinas disponibles en Colombia en pacientes adultos con dislipidemia con predominio de hipercolesterolemia que tienen indicación de tratamiento farmacológico, desde la perspectiva del sistema de salud colombiano.

\section{Material y métodos}

Se realizó un análisis de costo-efectividad basado en un modelo de Markov, con ciclos anuales, un horizonte temporal de cinco años, una tasa de descuento de $3 \%$ anual tanto para costos como para beneficios futuros, y la perspectiva de un tercero pagador (sistema de salud colombiano). Este modelo compara la efectividad, seguridad y costos de cinco medicamentos en pacientes que requieren terapia farmacológica (terapia intensiva o alta intensidad y terapia de intensidad moderada, según las recomendaciones actuales de manejo de la Asociación Americana del Corazón y adoptadas por la Guía de práctica clínica para la prevención, detección temprana, diagnóstico, tratamiento y seguimiento de las dislipidemias en la población mayor de 18 años. Convocatoria 563 de 2012 de Colciencias y el Ministerio de la Protección Social) (12). Para terapia moderada (atorvastatina, lovastatina, pravastatina, rosuvastatina y simvastatina) y en pacientes que requieren terapia intensiva (atorvastatina y rosuvastatina). Para realizar el modelo se empleó el programa en TreeAge Pro Healthcare ${ }^{\circledR}$ (Figura 1). Los desenlaces empleados fueron años de vida ajustados por calidad (AVAC) y disminución de eventos cardiocerebrovasculares (IAM, revascularización, ACV).

\section{Efectividad y seguridad}

Las probabilidades y los factores de riesgo de cada uno de los desenlaces, diabetes, muerte y abandono fueron extraídas de metaanálisis, ensayos clínicos aleatorizados y ensayos clínicos controlados. Las probabilidades y tasas se ajustaron a ciclos anuales para poder adaptarlas al modelo, ponderando por el número de participantes, como se muestra en la Tabla $1(7,11,13-52)$.

\section{Costos}

En el modelo, se incluyeron datos específicos para Colombia, costos del tratamiento, incluyendo el seguimiento, costos de consecuencias como la diabetes como efecto

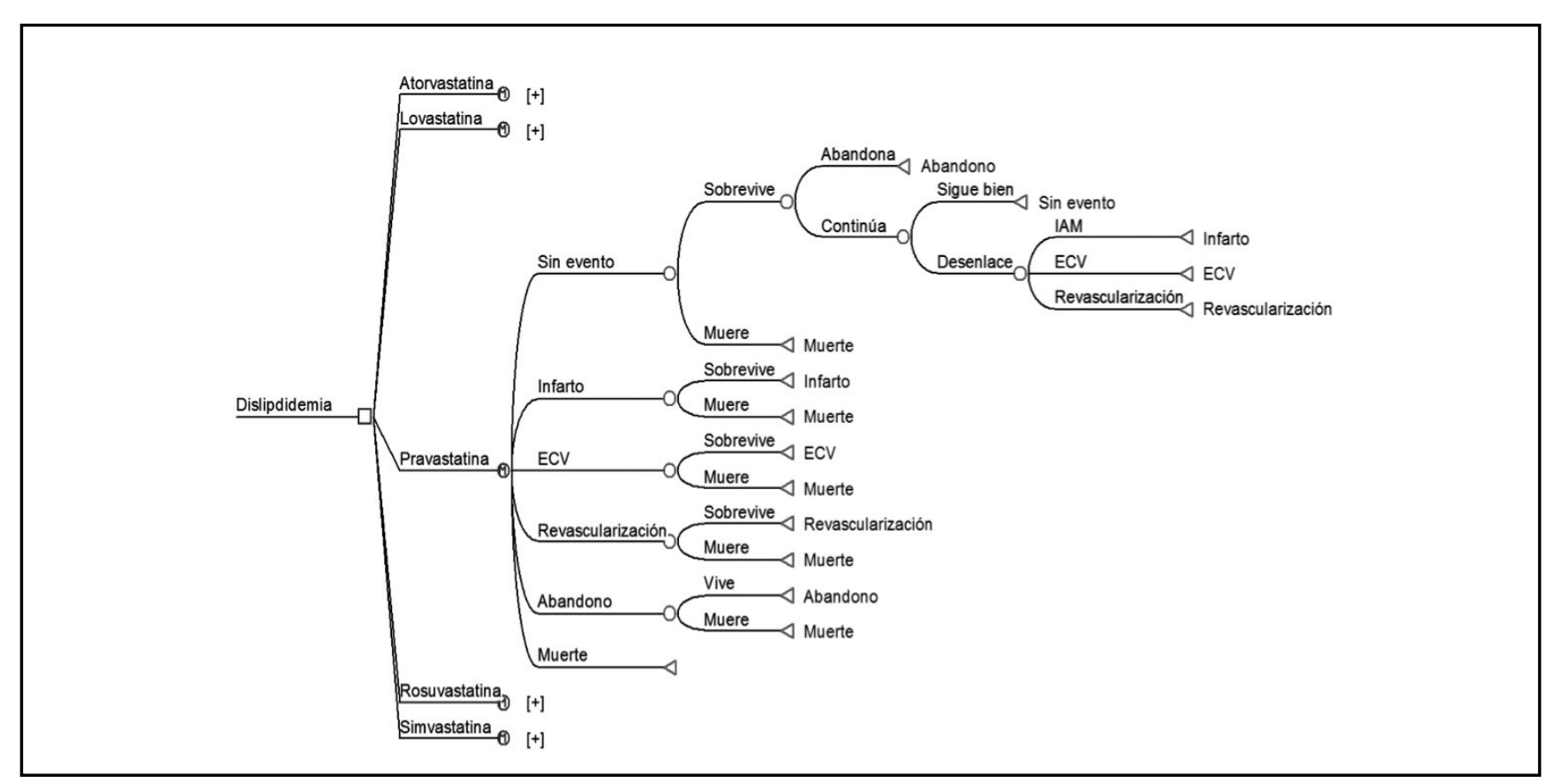

Figura 1. Modelo para pacientes en terapia moderada con estatinas. El modelo para terapia intensiva es similar, pero con sólo dos ramas (atorvastatina y rosuvastatina). Fuente: Elaborado por los autores. 
Tabla 1. Información sobre probabilidades y efectividades.

\begin{tabular}{|c|c|c|c|c|c|c|c|c|}
\hline Descripción & Eventos & Algún desenlace & ECV & IAM & Revascularización & Diabetes & Abandono & Muerte \\
\hline \multirow[t]{4}{*}{ Probabilidad } & Terapia intensidad moderada & $0.96 \%$ & $25.60 \%$ & $28.20 \%$ & $46.20 \%$ & $0.62 \%$ & $10.70 \%$ & $4.40 \%$ \\
\hline & Terapia de alta intensidad & $6.0 \%$ & $25 \%$ & $51.70 \%$ & $23.30 \%$ & & & \\
\hline & Atorvastatina & $0.63^{*}$ & 0.74 & 0.66 & 0.55 & 1.17 & 1.18 & 0.91 \\
\hline & Lovastatina & 0.70 & 0.62 & 0.62 & 0.76 & 0.98 & 0.91 & 0.95 \\
\hline \multirow[t]{3}{*}{ Factor de riesgo } & Pravastatina & 0.71 & 0.77 & 0.77 & 0.6 & 1.16 & 0.81 & 0.94 \\
\hline & Rosuvastatina & $0.61 *$ & 0.46 & 0.46 & 0.54 & 1.16 & 1.14 & 0.8 \\
\hline & Simvastatina & 0.89 & 0.75 & 0.96 & 0.93 & 1.1 & 0.89 & 0.87 \\
\hline
\end{tabular}

adverso asociado a las estatinas, así como los costos de cada uno de los desenlaces. Todos los costos están expresados en pesos colombianos de 2012. Para valorar los medicamentos, se consultaron los precios del Sistema Vademécum Med-Informática - Catálogo Farmacéutico Nacional (Sistema VMICFN) y SISMED de 2012, dada la perspectiva del estudio únicamente se tuvieron en cuenta los precios consignados en el canal institucional, para los medicamentos regulados se tomaron los precios reportados en las circulares emitidas por la Comisión Nacional de Precios de Medicamentos y Dispositivos Médicos (CNPMDM) como máximos; para el manejo de la enfermedad y valoración de desenlaces se emplearon las tarifas del Manual Tarifario del ISS - Acuerdo 256 de 2001, ajustadas al $35 \%$, este valor que fue sensibilizado al 25 y $48 \%$, y para el tratamiento de la diabetes, se empleó el costo enunciado por Tamayo (53) ajustado a 2012, con base en el índice de precios al consumidor (IPC) y la tasa de cambio de 2012 ( 1 USD = 2087.42 COP).

La estimación de costos de los medicamentos refleja el costo unitario ponderado de acuerdo con su participación en el mercado, sin diferenciar entre medicamentos con patente o genéricos. Para terapia moderada, las dosis promedio de recomendadas con atorvastatina son $40 \mathrm{mg} /$ día, lovastatina $30 \mathrm{mg} /$ día, pravastatina $40 \mathrm{mg} /$ día, simvastatina $40 \mathrm{mg} /$ día y rosuvastatina $20 \mathrm{mg} /$ día. Para terapia de alta intensidad, atorvastatina $80 \mathrm{mg} /$ día y rosuvastatina $40 \mathrm{mg} /$ día.

La Tabla 2 muestra los precios encontrados para cada una de las estatinas y el intervalo en el cual fluctúan los precios en el análisis de sensibilidad.

Finalmente, el costo de los desenlaces cardiocerebrovasculares refleja los costos de la atención ideal por medio de un caso tipo, definida por un consenso informal de expertos y teniendo en cuenta la frecuencia en ocurrencia para cada posible desenlace encontradas en la literatura científica (54-56). Conjuntamente, se validó esta información contra las facturas de pacientes diagnosticados con alguno de los desenlaces analizados. La estimación incorpora el número de días promedio de servicio, uso de recursos, promedio de consultas, estancia, medicamentos, procedimientos e insumos, esto se resumen en la Tabla 3.
Tabla 2. Costo unitario de las estatinas.

\begin{tabular}{|l|c|c|c|c|}
\hline Medicamento & Unidad & Base & Mínimo & Máximo \\
\hline Lovastatina & $\mathrm{mg}$ & $\$ 5.40$ & $\$ 1.00$ & $\$ 10.00$ \\
\hline Pravastatina & $\mathrm{mg}$ & $\$ 64.50$ & $\$ 5.00$ & $\$ 140.30$ \\
\hline Simvastatina & $\mathrm{mg}$ & $\$ 45.70$ & $\$ 4.10$ & $\$ 181.50$ \\
\hline Atorvastatina & $\mathrm{mg}$ & $\$ 7.50$ & $\$ 4.20$ & $\$ 158.50$ \\
\hline Rosuvastatina & $\mathrm{mg}$ & $\$ 288.90$ & $\$ 30.10$ & $\$ 469.10$ \\
\hline Fuente: SISMED. Precio ponderado Canal Institucional. \\
\hline
\end{tabular}

Tabla 3. Costo agrupado de los desenlaces.

\begin{tabular}{|l|c|c|c|}
\hline Desenlace & Valor & Mínimo & Máximo \\
\hline ECV & $\$ 5605191$ & $\$ 4965011$ & $\$ 6304107$ \\
\hline IAM & $\$ 9152054$ & $\$ 8217451$ & $\$ 9852266$ \\
\hline Revascularización & $\$ 5890008$ & $\$ 5448208$ & $\$ 6529013$ \\
\hline Rehabilitación ECV & $\$ 346221$ & $\$ 335076$ & $\$ 386343$ \\
\hline Rehabilitación IAM & $\$ 830513$ & $\$ 768994$ & $\$ 910489$ \\
\hline Fuente: Elaborado por los autores. \\
\hline
\end{tabular}

\section{Utilidades}

Dado que en Colombia no existe información válida sobre las ponderaciones de utilidad en los estados de salud considerados en el modelo, se emplearon las del registro Cost-Effectiveness Analysis Registry, del Center for the Evaluation of Value and Risk in Health del Institute for Clinical Research and Health Policy Studies (Tufts Medical Center).

De los estados de salud evaluados se encontraron 12 artículos, los cuales se agruparon por desenlace y posteriormente se realizó un promedio simple entre éstos. Finalmente, el valor promedio de utilidad para IAM fue 0.8 (57-60), ECV 0.595 (57, 58, 61, 62), revascularización 0.9085 (63-66), abandono 0,987 (7, 13, 57, 58, 62-66), diabetes $0.755(67,68)$ y para el paciente asintomático se realizó el supuesto de que estos pacientes se encuentran con salud perfecta, es decir 1 . 


\section{Resultados}

De acuerdo con lo expresado anteriormente, los resultados se presentan según el tipo de terapia.

\section{Terapia moderada}

El modelo de Markov arrojó para el horizonte temporal a cinco años, con una cohorte de mil pacientes y tasa de descuento de $3 \%$, que la rosuvastatina es la estrategia más efectiva en términos de AVAC (4.2417) pero a su vez la más costosa (10 millones de pesos), por otra parte, la pravastatina resultó ser una estrategia dominada por todas las estrategias.

Adicionalmente, se identificó que la atorvastatina es una estrategia costo-efectiva al compararla con la lovastatina, éstas mostraron costos muy similares, no obstante, la diferencia en efectividades favorece a la atorvastatina. Además su razón de costo-efectividad incremental no supera tres veces el Producto Interno Bruto (PIB) per cápita del año $2012 \$ 42863415$ (69).

Como se puede evidenciar, la rosuvastatina es la estrategia más efectiva pero su razón de costo-efectividad incremental \$171 347898 supera el umbral, y equivale a más de 22 veces el PIB per cápita colombiano de 2012. Para que esta estrategia alcance el umbral establecido, el costo mes de tratamiento tendría que reducir su valor actual en $84 \%$, para este horizonte temporal.

Igualmente el uso de lovastatina muestra un mayor número de muertes y desenlaces ${ }^{1}$ cardiocerebrovasculares, caso contrario ocurre con la rosuvastatina. La lovastatina, por cada 1000 pacientes produce menos abandonos, pues genera un aumento de tres muertes y ocho desenlaces con respecto a la atorvastatina, un incremento de 10 muertes y desenlaces en relación con la rosuvastatina, de una muerte y un desenlace con base en la pravastatina, y ocho muertes y un desenlace en el caso de la simvastatina. Por lo que se percibe que al evaluar otras medidas de efectividad la lovastatina no es tan efectiva como las otras estatinas.

\section{Terapia intensiva con estatinas}

Al igual que en terapia moderada, la rosuvastatina se asocia a una mayor efectividad con una ganancia en AVAC de 0.03 ; sin embargo, genera aumento en los costos de la terapia en 8 millones de pesos, en cuyo caso la razón de costo-efectividad incremental sería de $\$ 300$ millones aproximadamente. Por lo tanto, para este caso la atorvastatina es una estrategia costo-efectiva.

Evaluando otros desenlaces de interés, el tratamiento con rosuvastatina reduce en 11 casos de muertes, dos desenlaces cardiovasculares y ocho abandonos más que la atorvastatina. Donde el costo por evitar estas muertes con rosuvastatina equivaldría a \$8718 267998 y el costo evitado de los desenlaces \$1 341270462 .

Se realizaron análisis de sensibilidad univariado y probabilístico para evaluar la robustez de los resultados. El análisis

1 Desenlaces agrupa muertes, ECV, IAM y revascularización
Tabla 4. Efectividad y costo por paciente, obtenidos en el análisis de caso de referencia para tratamiento dislipidemia con terapia moderada con estatinas.

\begin{tabular}{|l|c|c|c|}
\hline Estrategia & Costo (\$) & Efectividad & $\begin{array}{c}\text { Razón de C/E } \\
\text { incremental (\$) }\end{array}$ \\
\hline Lovastatina & 851375 & 4.202 & \\
\hline Atorvastatina & 956087 & 4.213 & 9519273 \\
\hline Simvastatina & 1794824 & 4.2206 & 110360132 \\
\hline Pravastatina & 3702185 & 4.2046 & Dominada \\
\hline Rosuvastatina & 10059192 & 4.2417 & 171347898 \\
\hline Fuente: Elaborado por los autores. &
\end{tabular}

Tabla 5. Efectividad y costo por paciente con respecto a la atorvastatina, obtenidos en el análisis de caso de referencia para tratamiento dislipidemia con terapia intensiva con estatinas.

\begin{tabular}{|l|c|c|c|}
\hline Estrategia & Costo (\$) & Efectividad & $\begin{array}{c}\text { Razón de C/E } \\
\text { incremental (\$) }\end{array}$ \\
\hline Atorvastatina & 2816154 & 4.1479 & \\
\hline Rosuvastatina & 11534422 & 4.1776 & 293544377 \\
\hline \multicolumn{2}{|l}{ Fuente: Elaborado por los autores. } \\
\hline
\end{tabular}

univariado empleó los valores mínimos y máximos para cada uno de los parámetros y así saber el intervalo en el que se encontrará la razón de costo-efectividad incremental. Esto mostró que los costos mensuales de la lovastatina, atorvastatina y simvastatina tienen un mayor impacto sobre la razón de costo-efectividad, esto se debe a la alta variabilidad en los precios de estos medicamentos. Las otras variables tienen un impacto menor en los resultados del modelo.

El análisis de sensibilidad probabilístico se utilizó el método de simulación de Monte Carlo de primer orden, se asignaron distribuciones para cada uno de los parámetros, en relación con los costos, se supuso una distribución gamma para los desenlaces de ECV, IAM y revascularización; para otros costos, se empleó una distribución uniforme; para las utilidades, una distribución tipo beta, y para las probabilidades, distribución uniforme.

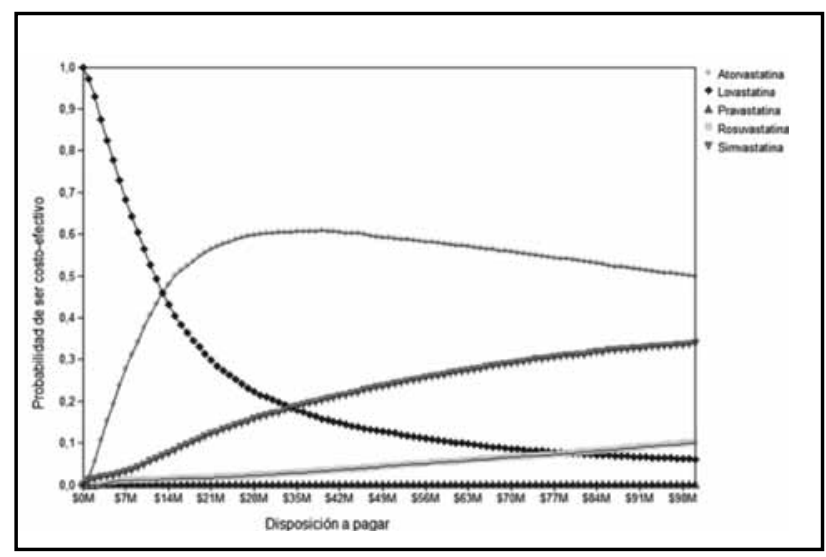

Figura 2. Curva de aceptabilidad para terapia moderada. 
Los resultados de estas simulaciones evidencian que no existe una alta variabilidad en los costos y AVAC de cada una de las estatinas, por ende los resultados son robustos a la incertidumbre y no modifican las conclusiones.

Las curvas de aceptabilidad confirman que la atorvastatina tiene mayor probabilidad de ser costo-efectiva que las otras alternativas, además que únicamente la lovastatina y la atorvastatina son estrategias costo-efectivas de acuerdo con la disposición a pagar del sistema de salud, donde para una disposición a pagar menor a 13 millones por AVAC ganado la lovastatina sería la estrategia más costo-efectiva.

\section{Discusión y conclusión}

En Colombia, a pesar de tener un gran número de diagnósticos poblacionales sobre enfermedades cardiovasculares no existen estudios que hayan abordado la costo-efectividad de sus intervenciones prioritarias. Este estudio se ha considerado relevante debido a que trata un tema que se considera importante tanto en mortalidad como en morbilidad en el país: la hipercolesterolemia es uno de los factores de riesgo modificables más comunes en la población, y su tratamiento con estatinas contribuye a la disminución de eventos cardiocerebrovasculares. Se espera que estos resultados apoyen las recomendaciones clínicas y, con un uso apropiado, generen una mejora significativa en la frecuencia y en la discapacidad de estas enfermedades en la población colombiana.

El análisis económico muestra que las diferencias absolutas entre las efectividades de las estatinas, independientemente del grupo poblacional, son relativamente pequeñas, especialmente en las personas de riesgo leve a moderado. Esta similitud tanto en efectividad como en seguridad lleva a que el costo del tratamiento sea un factor esencial en el análisis, convirtiéndolo a este estudio casi en un análisis de minimización de costos.

De acuerdo con la evidencia publicada, la rosuvastatina es la que genera mayor impacto en la reducción de los principales desenlaces cardiovasculares y en mortalidad en relación con las otras estatinas. Sin embargo, su costo actual hace que esa ganancia en las medidas de efectividad no se vea justificada, y ello hace que la atorvastatina sea la estrategia más costo-efectiva en Colombia.

La costo-efectividad de las estatinas en pacientes con trastornos del colesterol ha sido demostrada desde hace dos décadas en el Reino Unido (70), y ha sido ratificada para otros sistemas de salud (71) y validada incluso a través de revisiones sistemáticas de evaluaciones económicas (72). Aunque este trabajo tiene las limitaciones propias de los modelos económicos, incluyendo el empleo de probabilidades de transición y de utilidades derivadas de estudios foráneos, es la primera aproximación a su análisis en nuestro contexto. Algunos de los elementos de incertidumbre, como la adherencia de los pacientes a la terapia, la frecuencia de eventos adversos, o la influencia de las diferentes dietas regionales y estilos de vida de los colombianos ameritan más investigación.

\section{Fuentes de financiación y conflictos de interés}

El presente estudio fue elaborado en el marco de la Convocatoria 563 de 2012 de Colciencias y el Ministerio de la Protección Social.

D. Rosselli ha recibido honorarios como conferencista por parte de Astrazeneca, Merck Sharp \&Dohme, Pfizer y Bristol Myers Squibb, laboratorios involucrados en el desarrollo de estatinas. Ninguno de los otros autores declara conflicto de interés.

\section{Agradecimientos}

Agradecemos el apoyo del Departamento Administrativo de Ciencia, Tecnología e Innovación (Colciencias), el Ministerio de la Protección Socialy el Instituto de Evaluación Tecnológica en Salud -IETS.

\section{Referencias}

1. Instituto Nacional de Salud. Enfermedad cardiovascular: principal causa de muerte en Colombia. 2013. Disponible en: http://www.ins.gov.co/lineas-de-accion/ ons/boletin\%201/boletin_web_ONS/boletin_01_ONS.pdf

2. Messerli FH, Williams B, Ritz E. Essential hypertension. Lancet. 2007; 18;370: 591-603.

3. Canavan M, Emer McGrath, and Martin O'Donnell. Stroke. 6 th Ed. Philadelphia: Elsevier Health Scien; 2014.

4. Stamler J, Wentworth D, Neaton JD. Is relationship between serum cholesterol and risk of premature death from coronary heart disease continuous and graded? Findings in 356,222 primary screenees of the Multiple Risk Factor Intervention Trial (MRFIT). JAMA. 1986; 256: 2823-8.

5. Jousilahti P, Vartiainen E, Pekkanen J, Tuomilehto J, Sundvall J, Puska P. Serum cholesterol distribution and coronary heart disease risk observations and predictions among middle-aged population in eastern Finland. Circulation. 1998; 97: 1087-94.

6. Blood cholesterol and vascular mortality by age, sex, and blood pressure: a metaanalysis of individual data from 61 prospective studies with 55000 vascular deaths. Lancet. 2007; 370: 1829-39.

7. Taylor F, Huffman MD, Macedo AF, Moore THM, Burke M, Davey Smith G, et al. Statins for the primary prevention of cardiovascular disease.Cochrane Database Syst Rev. 2013 Jan;1:CD004816.

8. Van Halm VP, Nielen MMJ, Nurmohamed MT, van Schaardenburg D, Reesink HW, Voskuyl AE, et al. Lipids and inflammation: serial measurements of the lipid profile of blood donors who later developed rheumatoid arthritis. Ann Rheum Dis 2007; 66: 184-8.

9. Fletcher B, Berra K, Ades P, Braun LT, Burke LE, Durstine JL, et al. Managingabnormal blood lipids a collaborative approach. Circulation. 2005;112: 3184-209.

10. National Cholesterol Education Program (NCEP). Expert panel on detection, evaluation, and treatment of high blood cholesterol in adults (Adult Treatment Panel III) final report. Circulation 2002; 106: 3143-421.

11. Tonelli M, Lloyd A, Clement F, Conly J, Husereau D, Hemmelgarn B, et al. Efficacy of statins for primary prevention in people at low cardiovascular risk: a meta-analysis. Can MedAssoc J 2011; 183: E1189-E1202.

12. Ministerio de Salud y Protección Social, Colciencias, Pontificia Universidad Javeriana. Guía de práctica clínica para la prevención, detección temprana, diagnóstico, tratamiento y seguimiento de las dislipidemias en la población mayor de 18 años. 2014. Disponible en:http://www.iets.org.co/proyectos-en-curso/Paginas/ guia-dislipidemias.aspx

13. Ministerio de Salud y Protección Social, Colciencias, Universidad de Antioquia. Guía de práctica clínica para el síndrome coronario agudo. GPC-SCA; 2013. Disponible en: http://gpc.minsalud.gov.co/Documents/Guias-PDF-Recursos/SCA/ GPC_Comple_SCA.pdf

14. Ray KK, Seshasai S, Erqou S, et al. Statins and all-cause mortality in high-risk primary prevention: A meta-analysis of 11 randomized controlled trials involving 65229 participants. Arch Intern Med. 2010; 170: 1024-31.

15. Naci H, Brugts JJ, Fleurence R, Ades AE. Comparative effects of statins on major cerebrovascular events: a multiple-treatments meta-analysis of placebocontrolled and active-comparator trials. QJM. 2013; 106: 299-306.

16. Naci H, Brugts J, Ades T. Comparative tolerability and harms of individual statins: a study-level network meta-analysis of 246955 participants from 135 randomized, controlled trials. Circ Cardiovasc Qual Outcomes. 2013; 6: 390-9.

17. Naci H, Brugts JJ, Fleurence R, Tsoi B, Toor H,Ades AE. Comparative benefits of statins in the primary and secondary prevention of major coronary events and all-cause mortality: a network meta-analysis of placebo-controlled and activecomparator trials. Eur J Prev Cardiol. 2013; 20: 641-57. 
18. De Lemos JA, Blazing MA, Wiviott SD, Lewis EF, Fox KAA, White HD, et al. Early intensive vs a delayed conservative simvastatin strategy in patients with acute coronary syndromes: phase $\mathrm{Z}$ of the $\mathrm{A}$ to $\mathrm{Z}$ trial. JAMA. 2004 15; 292:130716.

19. Amarenco P, Bogousslavsky J, Callahan A 3rd, Goldstein LB, Hennerici M, Rudolph AE, et al. High-dose atorvastatin after stroke or transient ischemic attack. N Engl J Med. 2006; 355: 549-59.

20. Ridker PM, Danielson E, Fonseca FAH, Genest J, Gotto AM Jr, Kastelein JJP, et al. Rosuvastatin to prevent vascular events in men and women with elevated C-reactive protein. N Engl J Med. 2008; 359: 2195-207.

21. Cholesterol Treatment Trialists' (CTT) Collaborators. Efficacy and safety of cholesterol-lowering treatment: prospective meta-analysis of data from 90056 participants in 14 randomised trials of statins. Lancet. 2005; 366: 1267-78.

22. Nakamura H, Arakawa K, Itakura H, Kitabatake A, Goto Y, Toyota T, et al Primary prevention of cardiovascular disease with pravastatin in Japan (MEGA Study): a prospective randomised controlled trial. Lancet. 2006; 368: 1155-63.

23. Mihaylova B, Briggs A, Armitage J, Parish S, Gray A, et al. Lifetime cost effectiveness of simvastatin in a range of risk groups and age groups derived from a randomised trial of 20,536 people. BMJ. 2006; 333: 1145.

24. Bone HG, Kiel DP, Lindsay RS, Lewiecki EM, Bolognese MA, Leary ET, et al. Effects of atorvastatin on bone in postmenopausal women with dyslipidemia: a double-blind, placebo-controlled, dose-ranging trial. J Clin Endocrinol Metab. 2007; 92: 4671-7.

25. Sever PS, Dahlöf B, Poulter NR, Wedel H, Beevers G, Caulfield M, et al Prevention of coronary and stroke events with atorvastatin in hypertensive patients who have average or lower-than-average cholesterol concentrations, in the AngloScandinavian Cardiac Outcomes Trial--Lipid Lowering Arm (ASCOT-LLA): a multi centre randomised controlled trial. Lancet. 2003; 361: 1149-58.

26. Sattar N, Preiss D, Murray HM, Welsh P, Buckley BM, de Craen AJM, et al. Statins and risk of incident diabetes: a collaborative meta-analysis of randomised statin trials. Lancet. 2010; 375: 735-42.

27. Chan KL, Teo K, Dumesnil JG, Ni A, Tam J. Effect of Lipid lowering with rosuvastatin on progression of aortic stenosis: results of the aortic stenosis progression observation: measuring effects of rosuvastatin (ASTRONOMER) trial. Circulation. 2010; 121: 306-14.

28. Glynn RJ, Koenig W, Nordestgaard BG, Shepherd J, Ridker PM. Rosuvastatin for primary prevention in older persons with elevated C-reactive protein and low to average low-density lipoprotein cholesterol levels: exploratory analysis of a randomized trial. Ann Intern Med. 2010; 152: 488-496, W174.

29. Koenig W, Ridker PM. Rosuvastatin for primary prevention in patients with European systematic coronary risk evaluation risk $>=5 \%$ or Framingham risk $>20 \%$ : post hoc analyses of the JUPITER trial requested by European health authorities. Eur Heart J. 2011; 32: 75-83.

30. Albert MA, Glynn RJ, Fonseca FAH, Lorenzatti AJ, Ferdinand KC, MacFadyen JG, et al. Race, ethnicity, and the efficacy of rosuvastatin in primary prevention: the Justification for the Use of Statins in Prevention: an Intervention Trial Evaluating Rosuvastatin (JUPITER) trial. Am Heart J. 2011; 162: 106-114. e2.

31. Mora S, Glynn RJ, Hsia J, MacFadyen JG, Genest J, Ridker PM. Statins for the primary prevention of cardiovascular events in women with elevated highsensitivity C-reactive protein or dyslipidemia: results from the Justification for the Use of Statins in Prevention: An Intervention Trial Evaluating Rosuvastatin (JUPITER) and meta-analysis of women from primary prevention trials. Circulation. 2010; 121: 1069-77.

32. Collins R, Armitage J, Parish S, Sleigh P, Peto R. Heart Protection Study Collaborative Group. MRC/BHF Heart Protection Study of cholesterol-lowering with simvastatin in 5963 people with diabetes: a randomised placebo-controlled trial. Lancet. 2003; 361: 2005-16.

33. Binbrek AS, Elis A, Al-Zaibag M, Eha J, Keber I, Cuevas AM, et al. Rosuvastatin versus atorvastatin in achieving lipid goals in patients at high risk for cardiovascular disease in clinical practice: A randomized, open-label, parallelgroup, multicenter study (DISCOVERY Alpha study). Curr Ther Res. 2006; 67: 21-43.

34. Mohler ER 3rd, Hiatt WR, Creager MA. Cholesterol reduction with atorvastatin improves walking distance in patients with peripheral arterial disease. Circulation. 2003; 108: 1481-6.

35. Cannon CP, Braunwald E, McCabe CH, Rader DJ, Rouleau JL, Belder R, et al. Intensive versus moderate lipid lowering with statins after acute coronary syndromes. N Engl J Med 2004; 350: 1495-504.

36. Deedwania P, Stone PH, BaireyMerz CN, Cosin-Aguilar J, Koylan N, Luo D, et al. Effects of intensive versus moderate lipid-lowering therapy on myocardial ischemia in older patients with coronary heart disease: results of the Study Assessing Goals in the Elderly (SAGE). Circulation 2007;115: 700-7.

37. Nissen SE, Tuzcu EM, Schoenhagen P, Brown BG, Ganz P, Vogel RA, et al.
Effect of intensive compared with moderate lipid-lowering therapy on progression of coronary atherosclerosis: a randomized controlled trial. JAMA 2004; 291: 1071-80.

38. Pedersen TR, Faergeman O, Kastelein JJP, Olsson AG, Tikkanen MJ, Holme I, et al. High-dose atorvastatin vs usual-dose simvastatin for secondary prevention after myocardial infarction: the IDEAL study: a randomized controlled trial. JAMA 2005; 294: 2437-45.

39. Koren MJ, Hunninghake DB, ALLIANCE Investigators. Clinical outcomes in managed-care patients with coronary heart disease treated aggressively in lipid-lowering disease management clinics: the alliance study. J Am Coll Cardio 2004; 44:1772-9.

40. Fassett RG, Robertson IK, Ball MJ, Geraghty DP, Coombes JS. Effect of atorvastatin on kidney function in chronic kidney disease: a randomised doubleblind placebo-controlled trial. Atherosclerosis. 2010; 213: 218-24.

41. Knopp RH, d'Emden M, Smilde JG, Pocock SJ. Efficacy and safety of atorvastatin in the prevention of cardiovascular end points in subjects with type 2 diabetes: the Atorvastatin Study for Prevention of Coronary Heart Disease Endpoints in non-insulin-dependent diabetes mellitus (ASPEN).Diab Care. 2006; 29: 1478-85.

42. Wanner C, Krane V, März W, Olschewski M, Mann JFE, Ruf G, et al. Atorvastatin in patients with type 2 diabetes mellitus undergoing hemodialysis. $N$ Engl J Med 2005; 353: 238-48.

43. Crouse JR 3rd, Raichlen JS, Riley WA, Evans GW, Palmer MK, O'Leary DH, et al. Effect of rosuvastatin on progression of carotid intima-media thickness in low-risk individuals with subclinical atherosclerosis: the METEOR Trial. JAMA 2007; 297: 1344-53.

44. Gissi-HF Investigators, Tavazzi L, Maggioni AP, Marchioli R, Barlera S, Franzosi MG, et al. Effect of rosuvastatin in patients with chronic heart failure (the GISSI-HF trial): a randomised, double-blind, placebo-controlled trial. Lance 2008; 372: 1231-9.

45. MRC/BHF Heart Protection Investigators. MRC/BHF Heart Protection Study of cholesterol-lowering therapy and of antioxidant vitamin supplementation in a wide range of patients at increased risk of coronary heart disease death: early safety and efficacy experience. Eur Heart $J$ 1999; 20: 725-41.

46. Armitage J, Bowman L, Wallendszus K, Bulbulia R, Rahimi K, et al. Study of the Effectiveness of Additional Reductions in Cholesterol and Homocysteine (SEARCH) Collaborative Group, Intensive lowering of LDL cholesterol with 80 $\mathrm{mg}$ versus $20 \mathrm{mg}$ simvastatin daily in 12,064 survivors of myocardial infarction: a double-blind randomised trial. Lancet 2010; 376: 1658-69.

47. Davidson MH, Palmisano J, Wilson H, Liss C, Dicklin MR. A multicenter, randomized, double-blind clinical trial comparing the low-density lipoprotein cholesterol-lowering ability of lovastatin 10,20 , and $40 \mathrm{mg} / \mathrm{d}$ with fluvastatin 20 and $40 \mathrm{mg} / \mathrm{d}$. ClinTher. 2003; 25: 2738-53.

48. Gentile S, Turco S, Guarino G, Sasso CF, Amodio M, Magliano P, et al Comparative efficacy study of atorvastatin vs simvastatin, pravastatin, lovastatin and placebo in type 2 diabetic patients with hypercholesterolaemia. Diab Obes Metab 2000; 2: 355-62.

49. Andrews TC, Ballantyne CM, Hsia JA, Kramer JH. Achieving and maintaining national cholesterol education program low-density lipoprotein cholesterol goals with five statins. Am J Med 2001; 111: 185-91.

50. Sato H, Kinjo K, Ito H, Hirayama A, Nanto S, Fukunami M, et al. Effect of early use of low-dose pravastatin on major adverse cardiac events in patients with acute myocardial infarction: the OACIS-LIPID Study. Circ J 2008; 72: 17-22.

51. Asselbergs FW, Diercks GFH, Hillege HL, van Boven AJ, Janssen WMT, Voors AA, et al. Effects of fosinopril and pravastatin on cardiovascular events in subjects with microalbuminuria. Circulation 2004; 110: 2809-16.

52. Mancia G, Parati G, Revera M, Bilo G, Giuliano A, Veglia F, et al. Statins, antihypertensive treatment, and blood pressure control in clinic and over 24 hours: evidence from PHYLLIS randomised double blind trial. BMJ 2010; 340: c1197.

53. Tamayo D. Diabetes en Colombia: costos asociados con su cuidado. 2013;1-8 Disponible en:http://www.odc.org.co/files/Diabetes_en_Colombia_-_Costos_asociados_con_su_cuidado.pdf

54. Hong K-S, Bang OY, Kim JS, Heo JH, Yu K-H, Bae H-J, et al. Stroke Statistics in Korea: Part II. J Stroke 2013; 15: 67-77.

55. Meza Bejarano LY, Amaya Gonzalez P, Rodriguez JH. Causas de no trombolisis en ataque cerebrovascular. Acta Neurol Colomb 2013;29:4-9. Disponible en: http:/www.acnweb.org/es/acta-neurologica/volumen-29-2013/142-volumen29-no-1/817-causas-de-no-trombolisis-en-ataque-cerebrovascular.htm

56. Guias de atención Sociedad Colombiana de Cardiología y Cirugía Cardiovascular. Disponible en: http://scc.org.co/blog/category/guias-de-atencion/

57. Guerriero C, Cairns J, Roberts I, Rodgers A, Whittaker R, Free C. The cost-effectiveness of smoking cessation support delivered by mobile phone tex messaging. Health Econ Prev Care 2013; 14: 789-97.

58. Gada H, Desai MY, Marwick TH. Cost-effectiveness of computed tomographic 
angiography before reoperative coronary artery bypass grafting: a decision-analytic model. Circ Cardiovasc Qual Outcomes 2012; 5: 705-10.

59. You JHS, Tsui KKN, Wong RSM, Cheng G. Cost-effectiveness of dabigatran versus genotype-guided management of warfarin therapy for stroke prevention in patients with atrial fibrillation. PloS One. 2012; 7(6): e39640.

60. Lee S, Anglade MW, Meng J, Hagstrom K, Kluger J, Coleman CI. Costeffectiveness of apixaban compared with aspirin for stroke prevention in atrial fibrillation among patients unsuitable for warfarin. Circ Cardiovasc Qual Outcomes 2012; 5: 472-9.

61. McCullagh L, Walsh C, Barry M. Value-of-information analysis to reduce decision uncertainty associated with the choice of thromboprophylaxis after total hip replacement in the Irish healthcare setting. Pharmaco Econ 2012; 30: 941-59.

62. Athanasakis K, Igoumenidis M, Karampli E, Vitsou E, Sykara G, Kyriopoulos J. Cost-effectiveness of varenicline versus bupropion, nicotine-replacement therapy, and unaided cessation in Greece. Clin Ther 2012; 34: 1803-14.

63. Menown I, Montalescot G, Pal N, Fidler C, Orme M, Gillard S. Enoxaparin is a cost-effective adjunct to fibrinolytic therapy for ST-elevation myocardial infarction in contemporary practice. Adv Ther 2010; 27: 181-91.

64. Rosen VM, Taylor DCA, Parekh H, Pandya A, Thompson D, Kuznik A, et al. Cost effectiveness of intensive lipid-lowering treatment for patients with congestive heart failure and coronary heart disease in the US. Pharmaco Econ 2010; 28: 47-60.

65. Wagner M, Lindgren P, Merikle E, Goetghebeur M, Jönsson B. Economic evaluation of high-dose $(80 \mathrm{mg} /$ day $)$ atorvastatin treatment compared with standard-dose $(20 \mathrm{mg} /$ day to $40 \mathrm{mg} /$ day $)$ simvastatin treatment in Canada based on the Incremental Decrease in End-Points Through Aggressive Lipid-Lowering (IDEAL) trial. Can J Cardiol 2009; 25: e362-369.

66. Lindgren P, Buxton M, Kahan T, Poulter NR, Dahlöf B, Sever PS, et al. The lifetime cost effectiveness of amlodipine-based therapy plus atorvastatin compared with atenolol plus atorvastatin, amlodipine-based therapy alone and atenolol-based therapy alone: results from ASCOT1.Pharmaco Econ 2009; 27: 221-30.

67. Hoerger TJ, Schillie S, Wittenborn JS, Bradley CL, Zhou F, Byrd K, et al. Cost-effectiveness of hepatitis B vaccination in adults with diagnosed diabetes. Diab Care 2013; 36: 63-9.

68. Garcia-Ruiz AJ, Perez-Costillas L, Montesinos AC, Alcalde J, Oyaguez I, Casado MA. Cost-effectiveness analysis of antipsychotics in reducing schizophrenia relapses. Health Econ Rev 2012; 2: 8.

69. Banco de la República de Colombia. PIB total y por habitante .2014. Disponible en: http://www.banrep.gov.co/es/pib

70. Pharoah PD1, Hollingworth W. Cost effectiveness of lowering cholesterol concentration with statins in patients with and without pre-existing coronary heart disease: life table method applied to health authority population. BMJ 1996; 312: 1443-8.

71. Pletcher MJ1, Lazar L, Bibbins-Domingo K, Moran A, Rodondi N, CoxsonP,et al. Comparing impact and cost-effectiveness of primary prevention strategies for lipid-lowering. Ann Intern Med 2009; 150: 243-54.

72. Ward S1, Lloyd Jones M, Pandor A, Holmes M, Ara R, Ryan A, et al. A systematic reviewand economic evaluation of statins for the prevention of coronary events.HealthTechnolAssess. 2007;11(14):1-160. and economic evaluation of statins for the prevention of coronary events. Health Technol Assess 2007; 11(14): $1-160$. 